\title{
Vulnerability to Natural Disasters and Insurance: Insights from the Italian Case
}

\author{
Francesco De Masi and Donatella Porrini * \\ Dipartimento di Scienze dell'Economia, Università del Salento, Via per Monteroni, 73100 Lecce, Italy; \\ francesco.demasi@unisalento.it \\ * Correspondence: donatella.porrini@unisalento.it; Tel.: +39-0832-29-8776
}

Received: 5 January 2018; Accepted: 24 May 2018; Published: 5 June 2018

\begin{abstract}
The aim of this article is to analyze the concept of "vulnerability" to natural disasters, focusing on the specific case of the Italian earthquake insurance. In this sense, we examine in detail the vulnerability definition and its relevance for citizens and for insurance companies. Then we investigate the Italian insurance market characterized by a very low penetration of natural disasters insurance and the potential effects of a recent Government initiative called "Sisma Bonus". The idea is that its technical content, in terms of a specific definition of vulnerability, may contribute to developing a better consciousness about vulnerability and a larger diffusion of insurance products.
\end{abstract}

Keywords: vulnerability; insurance; natural disasters; Italy

JEL Classification: G22; G28; H24; Q54

\section{Introduction}

At a global level, in 2016 there were 327 disaster events $^{1}$, and this value reflects the tendency of a gradual but significant increase in the number of catastrophes in the last 40 years ${ }^{2}$.

At a European level, the European Environment Agency (EEA) outlines a dramatic situation where the frequency of environmental disasters has been raising significantly ${ }^{3}$. The events, considered by the Agency, are natural calamities such as earthquakes, seaquakes, landslides, floods; that is to say a set of natural phenomena different from each other, which occurred in different regions, according to the environmental characteristics.

For this reason, there has been an increase in the exposition of the territory and the economy towards disaster risks and the role of insurance products is becoming more and more relevant, because they offer an ex-post compensation for possible damages, but also because they incentivize the ex-ante arrangement of appropriate precautionary measures, the so-called risk mitigation (Porrini and Schwarze 2014).

In this respect, Italy is a country particularly exposed towards catastrophic events, based on the configuration of the land, but at the same time is a country where there is an insufficient penetration of insurance policies.

The historical analysis confirms this consideration: only in the last 150 years, a seismic disaster occurred on average every 4-5 years; in the same period, more than 2800 landslides and a particular elevated number of floods occurred in the country (Guidobboni and Valensise 2013).




In particular, focusing the attention on the most recent occurrences, two earthquakes have hit Italy. The first refers to May 2012 when two seismic swarms in Emilia Romagna caused damages for about 13.3 billion of Euros and insurance losses of around 1.24 billion Euro (corresponding to a percentage near the $9.3 \%$ of the total economic losses). The second, instead, refers to the catastrophic events in the Center of the Country starting in August 2016 in the area of Amatrice, Accumuli and Norcia. In this case the economic damages were about 7.1 billion Euro, while the insurance losses were around 106.5 million Euro (corresponding to only the $1.5 \%$ of the total economic losses) 4 .

These events show the high vulnerability of the national territory, of the historical heritage and the architectural buildings. The high amount of economic losses attests the described scenario and underlines the necessity of the definition of a correct contrast strategy.

From these first reflections, also emerges the limited recourse to insurance as a financial tool to cover the damages derived from natural disasters. This is in connection with the fact that in Italy, it is generally expected that, if a certain natural disaster happened, the State will intervene punctually with assistance measures aimed at the determination of aids to the population involved and thereafter the distribution of public economic resources for the reconstruction (Porrini 2016).

This article therefore intends to investigate the reasons of this condition, trying to examine in detail the vulnerability concept and its relevance for citizens and for insurance companies. In this sense, the next section provides an in-depth study about the vulnerability concept, by analyzing the theoretical and applied aspects. The second focuses on the relevance of that concept and on its influence on the demand and supply of insurance policies, focusing on the Italian scenario. Finally, the third and last section considers the Italian Government intervention that introduced the so called "Sisma Bonus" with the goal of building a national plan of prevention of seismic events.

\section{Vulnerability: Theoretical and Applied Aspects}

Referring to the traditional definition of the risk concept ${ }^{5}$, we intend to focus on a specific element: Vulnerability. Around it, the evaluations here realised try to analyse all the aspects directly related, in order to contribute to the existing body of the knowledge.

"Vulnerability" can be defined as the characteristic of a single person or a group that influence their capacity to anticipate, cope with, resist and recover from the impact of a natural hazard (an extreme natural event or process) (Wisner et al. 2003). In addition to people, it is also necessary to extend the concept to buildings and infrastructures. In this case, vulnerability expresses the percentage of the economic value that can be impaired from the occurrence of the considered event (Canuti and Casagli 1994). Furthermore the specific features of the territory and its characteristics are important, because they enable to calculate the vulnerability also from a geological point of view.

The three differ definitions, quoted above, are represented in Figure 1.

Figure 1 sums up the three main aspects that we have to consider in order to provide a complete and exhaustive definition of the vulnerability concept: The social aspect, the economic aspect, and the physical and environmental aspect. These three elements particularly interconnected one with one another, stemming from the descriptions previously carried out. Consequently, in order to reach a complete and true understanding, it is necessary to adopt an interdisciplinary approach that allows to reach an overall view.

4 PERILS (2017).

5 Following the traditional definition: Risk $=$ Hazard $\times$ Exposure $\times$ Vulnerability

"Hazard" refers to the probability that a specific event, potentially dangerous, with a high intensity, can occur in a specific area, in a specific time; "exposure" refers to the exposed elements, that is to say population, properties, economical activities, public services, environmental and cultural goods. It can be identified through the number of units (for example the number of monuments), the economic value or the percentage of expected loss; "vulnerability" will be detailed descripted in this section. (Canuti and Casagli 1994; Einstein 1988; Fell 1994; Varnes 1984). 




Figure 1. Vulnerability aspects.

In fact, the definitions that stem from the vulnerability concept could be very different due to the extent of the elements and of the points of view from which it can be analyzed.

For this reason, it is important to remember one of the best well-known definitions. It is the one of the UNISDR (United Nations Office for Disaster Risk Reduction) that defines vulnerability as "the conditions determined by physical, social, economic and environmental factors or process, which increase the susceptibility of a community to the impact of hazards" ${ }^{\prime \prime}$.

In reality, this kind of definitions identifies a static situation, because it describes the conditions of a population, an immovable property or natural resource. As a function of corrective actions, indeed, a lower level of vulnerability is possible. Therefore, a dynamic profile of the vulnerability concept emerges. In this field, the UNDP (United Nations Development Programme) defines vulnerability as a human condition or process resulting from physical, social, economic and environmental factors, which determine the likelihood and scale of damage from the impact of a given hazard ${ }^{7}$.

In this regard, we may find a starting point in the World Conference on Natural Disaster Reduction (Yokohama, Japan, May 1994), where the guidelines for an improvement in this sense were indicated. The principal elements, that were recalled, referred mainly to the spreading of a culture of prevention and a better awareness towards these themes from private citizens, authorities and institutions (so-called Yokohama Strategy).

In order to reach this goal, there are essentially two fields that require more efforts: the definition of adequate contrast policies towards catastrophic events and a punctual regulation of the rules in terms of respect for the environment and the construction of buildings and infrastructures. In this perspective the insurance solution represents a useful strategy to be adopted because it allows obtaining to obtain advantages both in a perspective of damages coverage, but also in the capability of incentivizing the construction of new buildings and the realization of reinforcing works in line with prevention regulation. It is necessary, therefore, to act, above all, in these fields in order to improve the national system. 


\section{Vulnerability and the Role of Insurances}

The definition of the vulnerability concept is fundamental in the study and in-depth analysis of insurance models. It represents a key element in the determination and development of a system that should be able to efficiently contrast the environmental disasters damages.

These considerations are true not only for citizens who seek an insurance policy for these events, but also for the insurance companies that have to take into account them in order to set fair premiums. Both points of view aim for the determination of a specific territory's riskiness and for the appropriateness of the building regulations adopted for the construction. In other words, what is important to focus on is the capability of a specific element (population, buildings, infrastructure, activities, and natural resources) to withstand the effects depending on the event intensity.

\subsection{How Vulnerability Influences the Demand of Insurance Products: The Italian Case}

Focusing on the Italian scenario, the catastrophic events that have hit the Center of Italy since the August 2016, once again have drawn the attention to the extreme vulnerability of national territory, highlighting that the Italian cultural heritage is not able to withstand an earthquake, even it is not particularly high intensity.

Although the basic ideas are similar, the relevance of these considerations is different for insurance companies or for private citizens. Starting from the latter, according to a recent market research of GSK Eurisko the majority of Italian families does not think they are particularly exposed to the risk of catastrophic events 8 .

In particular, data show the following situation:

- $17 \%$ believe that their own homes are exposed to catastrophic risks;

- $63 \%$ believe that their own homes are not exposed to catastrophic risks;

- $20 \%$ do not know.

This research shows that about the $83 \%$ of the population does not admit they are part of a segment characterized by a particularly high exposition and, for this reason, does not think necessary to purchase an insurance policy that covers a risk that is not considered real. The direct consequence of those considerations is that only one house out of 50 in Italy is covered by an insurance policy against the natural disasters risk, earthquake, flood or both ${ }^{9}$. These data must be associated with a substantial complexity and uncertainty relating to the construction materials used and their deterioration level. These are evaluations that draw greatly on buildings vulnerability, above all if they are quite old (D'Ayala et al. 2006).

Furthermore, we have also to consider the consciousness that if a natural disaster comes true, the Government reply would be prompt in order to take the necessary steps to help the population affected. This attitude is widely spread in Italy but, indeed, when faced with a catastrophic event, the Government sets different measures according to the different situations.

The recent events in the Center of Italy, where there have been several and repeated seismic activities in Amatrice, Accumuli and Norcia are emblematic. An approach close to the one described was adopted after these events. Analyzing other environmental disasters, we can see how this kind of contrast strategy represents the historical way the Italian Governments has faced these situations, implementing ex-post public interventions.

This kind of interventions is not considered compulsory by law, but is, instead, recognized by the Institutions, which must operate to contrast the natural disasters damages at a national level. Currently, it is a consolidated procedure through which the Government, after every natural disaster, issues specific measures, above all taxes, in order to raise economic funds to carry out aid, to reactivate 
traffic structures, to indemnify the damages suffered by public Authorities, private citizens and firms (Porrini 2010).

The described scenario has contributed to a limited diffusion of private solutions and it is an evaluation necessary in order to examine the origin of the $83 \%$ of the population who do not believe they are exposed to natural disasters. Furthermore, there is another relevant element to be evaluated. The penetration of insurance coverage for catastrophic events is very limited: $95 \%$ of policies do not show any insurance extension, as we can see in Table 1.

Table 1. Policies extension to natural disasters.

\begin{tabular}{ccccc}
\hline $\begin{array}{c}\text { Extension to } \\
\text { Natural Disasters }\end{array}$ & Policies Number & $\begin{array}{c}\text { Distribution \% } \\
\text { Policies Number }\end{array}$ & $\begin{array}{c}\text { Insurance Values } \\
\text { (mln of Euro) }\end{array}$ & $\begin{array}{c}\text { Distribution \% } \\
\text { Insurance Values }\end{array}$ \\
\hline No Extension & $8,253,990$ & $95.0 \%$ & $3,206,693$ & $95.9 \%$ \\
Only earthquake risk & 188,364 & $2.2 \%$ & 74,061 & $2.2 \%$ \\
Only flood risk & 184,963 & $2.1 \%$ & 39,030 & $1.2 \%$ \\
Both & 62,074 & $0.7 \%$ & 23,793 & $0.7 \%$ \\
TOTAL & $8,689,391$ & $100.0 \%$ & $3,343,576$ & $100.0 \%$ \\
\hline
\end{tabular}

Our elaboration of data from: Ania (2017).

Table 1 shows in the detail the limited extension to natural disasters of insurance policies in the market, according to the data spread on 30 September 2016. Therefore the data underline the limited consciousness of citizens about their vulnerability and their exposition to these events: to strengthen this argument, it is estimated that only 610,000 houses out of 31.2 million (according to the latest data published by ISTAT-The Italian National Institute of Statistics) are insured, more or less $2 \%$.

These data are in clear contrast with the Italian territory configuration, a country historically exposed towards these events. In particular, it is clear that about the $78 \%$ of Italian houses are characterized by a high or a medium-high risk with reference to earthquake and hydrogeological phenomena (Farina 2017).

For this reason, the limited consciousness of citizens is not justified in terms of exposition and vulnerability. However, the reasons we must consider here are: The limited insurance culture; the widespread certainty about the existence of a kind of "certain compensation" from the Government, and the underestimation of the risk perception because of the rarity of these events, even though they are of high intensity (Ronchi 2017).

\subsection{How Vulnerability Influences the Supply of Insurance Products}

On the contrary, the importance attached to vulnerability concept by the insurance companies is different. When they implement actuarial models to supply their products, they deeply analyze the territory and the buildings features, as well as their exposition towards these events.

The main phases and activities that each company follows in this sector can be summarized in four key points (Weymann 2015), as in Figure 2.



Figure 2. Evaluation steps. 
The first element the companies need to consider is the risk identification they have to cover financially. A deep analysis about the event to be insured is necessary, finding all the specific characteristics.

The second is the evaluation in terms of repetitiveness and proportions of the damages that could derive. In this specific step, the vulnerability concept plays a very important role. Without an accurate study of these elements, the definition of a correct financial strategy will undoubtedly be misleading and barely available for adoption. All the insurance companies have to necessarily analyze in the detail the territorial features, in relation to the exposition towards the catastrophic event considered. These evaluations represent the starting point for the construction of a reliable model of reference and for the determination of an insurance product that can be offered to the market with success.

The third and the fourth phases are mainly based on the identification of a preventive strategy and on the capability of being able to manage the residual risk. Under the first aspect, it is important to underline the relevance of insurance not only in an "ex-post" scenario in respect to the catastrophic event, but also in an "ex-ante" one trying, in other words, to incentivize the construction of new buildings or the fulfilment of consolidation works following the prevention regulation. Therefore this condition generally improves the exposition and the vulnerability to natural disasters. Specifically, insurance companies can provide economic incentives for risk prevention measures: By fixing the premium in function of the risk, they can motivate contractors to personally intervene in order to make their own properties less vulnerable ${ }^{10}$.

The analysis above is in line with what we have affirmed at the beginning of this paragraph about the different perception towards the vulnerability. In fact, vulnerability is a structural element for insurance companies differently from the citizens, who do not seem to have a correct knowledge of it.

\subsection{The Evolution of the Vulnerability Concept in an Insurance Prospective}

The vulnerability concept, repeatedly quoted in this article, is a particularly broad topic and can be analyzed from different point of view in relation to the elements taken into consideration. A territory, for example, can be exposed to a certain event due to its morphological configuration, as well as the events that has occurred over the years.

Focusing our attention on the earthquake risk in Italy, the above map (Figure 3) locates the area characterized by a seismic dangerousness along the national territory, with colors more or less intense in connection to more or less elevated exposition.

Nevertheless it is important to underline that this is not sufficient to identify a high or low exposition of a certain building or a single house. Where, in fact, buildings were constructed following the prevention regulation that would secure the property from potential disasters, then it would be able to mitigate the vulnerability, despite any features of the territory. These are considerations that directly refer to the definition of vulnerability. With respect to the three profiles from which the vulnerability concept stems, the elements that contribute to its relevance are different.

Looking at the territorial characteristics, focusing the attention on the aspect linked to the constructions quality, the main elements that influence the vulnerability can be summarized as the followings: planning errors, construction errors, materials age. These factors have a direct incidence to the exposition size of a certain building, because they draw on the resistance capability in respect to a catastrophic event. They can be identified in three different moments:

I. Pre-consolidation: Concentrating resources to make a consolidation intervention;

II. Pre-event: Identifying the most vulnerable elements;

III. Post-event: Localizing damages.

10 European Commission (2013). 


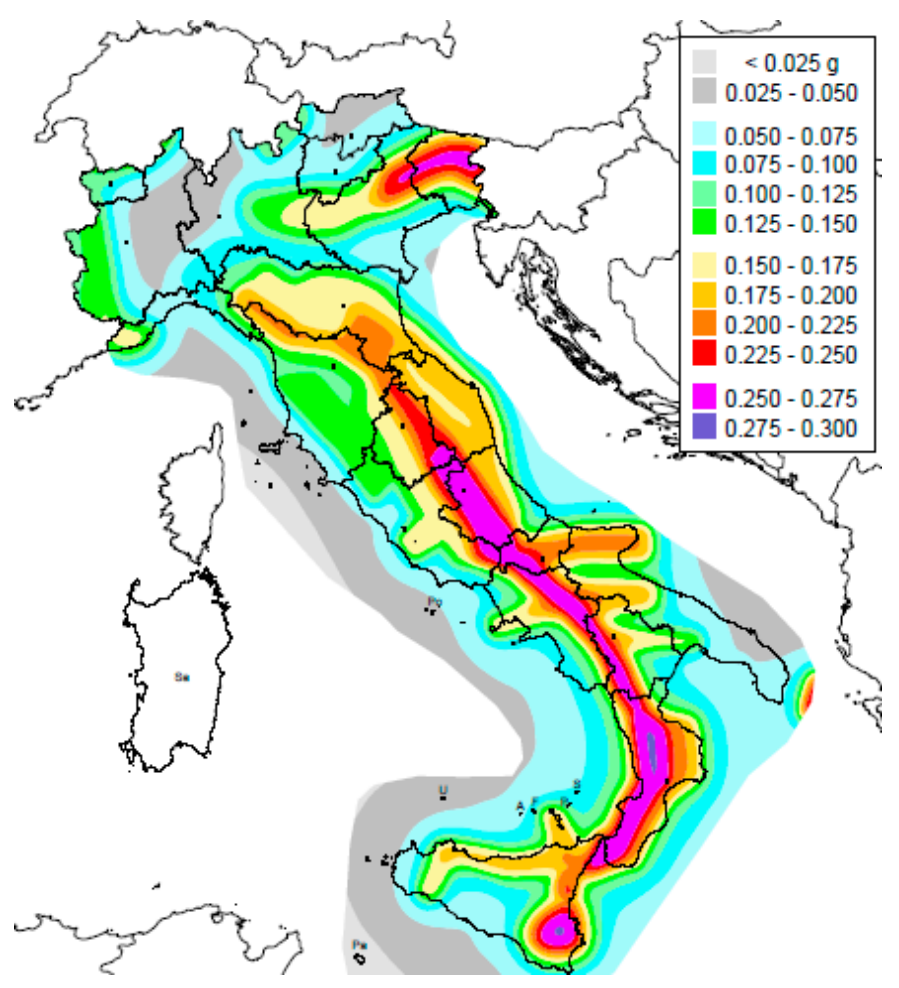

Figure 3. Map of seismic dangerousness along the national territory. Source: Italian National Institute of Geophysics and Volcanology, April 2004.

\section{The Italian Case of "Sisma Bonus"}

The Italian Government plans to move in the direction to stimulate the citizens' consciousness about their exposition, as well as reducing buildings vulnerability.

Focusing carefully on this new strategy, it has recently approved, in the "Legge di Stabilità 2017"11, a national plan for the evaluation and classification of the buildings seismic exposition and a relative prevention plan, with the goal of improving risk mitigation. It is an action particularly relevant because it fits perfectly in a scenario to incentivize a higher perception and consciousness of its own vulnerability, given that measuring vulnerability, indeed, is a fundamental passage for an effective risk reduction and for the promotion of a culture of disaster resilience (Birkmann 2006).

In the Ministerial Decree, all the characteristics of the new system are described in detail: the guidelines, that allow for a classification of buildings according to the seismic risk, are highlighted, as well as the way to certify the efficiency of the interventions realized by qualified professionals.

Along with this measure, there is a system of tax concession that allows spreading it in a fast and effective way. It is composed by a set of tax allowances that are recognized in the case that, after having carried out adjustments on the house, there is an improvement of one, two or more seismic risk classes.

Moreover, the legislative regulation provides for a system based on the identification of eight risk classes. Placed in ascending order, they are indicated as follows: $\mathrm{A}^{+}, \mathrm{A}, \mathrm{B}, \mathrm{C}, \mathrm{D}, \mathrm{E}, \mathrm{F}, \mathrm{G}$. The definition of the risk class, that marks a home, is carried out through two methods alternatively: the conventional and the simplified method.

The first is a universal method, that is to a say a method that can be used with all the construction typologies, differently from the second that can be used only in case of masonry construction.

11 Ministerial Decree number 65, 07/03/2017. 
Both methods allow for a preventive evaluation of its current class, as well as a further analysis of the new class determination, based on possible adaptation works.

The selection of the correct class of affiliation needs accurate evaluations. A first parameter to consider is the PAM ("Perdita Annua Media attesa"—expected average annual loss), that is to say the annual allocation of the costs for the damages deriving from future seismic phenomena, expressed as a percentage $^{12}$.

Referring to the conventional method, a first classification takes place regarding to the value of the PAM, in relation to the parameters indicated in Table 2.

Table 2. PAM Index.

\begin{tabular}{cc}
\hline Expected Average Annual Loss (PAM) & PAM Class \\
\hline PAM $\leq 0.50 \%$ & $\mathrm{~A}^{+}$PAM \\
$0.50 \%<$ PAM $\leq 1.0 \%$ & $\mathrm{~A}_{\mathrm{PAM}}$ \\
$1.0 \%<\mathrm{PAM} \leq 1.5 \%$ & $\mathrm{~B}_{\mathrm{PAM}}$ \\
$1.5 \%<\mathrm{PAM} \leq 2.5 \%$ & $\mathrm{C}_{\mathrm{PAM}}$ \\
$2.5 \%<\mathrm{PAM} \leq 3.5 \%$ & $\mathrm{D}_{\mathrm{PAM}}$ \\
$3.5 \%<\mathrm{PAM} \leq 4.5 \%$ & $\mathrm{E}_{\mathrm{PAM}}$ \\
$4.5 \%<\mathrm{PAM} \leq 7.5 \%$ & $\mathrm{~F}_{\mathrm{PAM}}$ \\
$7.5 \% \leq \mathrm{PAM}$ & $\mathrm{G}_{\mathrm{PAM}}$ \\
\hline
\end{tabular}

Source: Ministerial Decree number 65, 07/03/2017.

Alongside this evaluation, another parameter is usually adopted, namely the IS- $\mathrm{V}^{13}$ security index of each home, in relation to which it is possible to obtain a further classification.

As Table 3 shows, this index involves a building classification in seven risk classes, differently from the PAM parameter that considers eight classes.

Table 3. Security index.

\begin{tabular}{cc}
\hline SECURITY INDEX & IS-V CLASS \\
\hline $100 \%<\mathrm{IS}-\mathrm{V}$ & $\mathrm{A}^{+}$IS-V \\
$80 \% \leq \mathrm{IS}-\mathrm{V}<100 \%$ & $\mathrm{~A}_{\mathrm{IS}-\mathrm{V}}$ \\
$60 \% \leq \mathrm{IS}-\mathrm{V}<80 \%$ & $\mathrm{~B}_{\mathrm{IS}-\mathrm{V}}$ \\
$45 \% \leq \mathrm{IS}-\mathrm{V}<60 \%$ & $\mathrm{C}_{\mathrm{IS}-\mathrm{V}}$ \\
$30 \% \leq \mathrm{IS}-\mathrm{V}<45 \%$ & $\mathrm{D}_{\mathrm{IS}-\mathrm{V}}$ \\
$15 \% \leq \mathrm{IS}-\mathrm{V}<30 \%$ & $\mathrm{E}_{\mathrm{IS}-\mathrm{V}}$ \\
IS-V $\leq 15 \%$ & $\mathrm{~F}_{\mathrm{IS}-\mathrm{V}}$ \\
\hline Source: Ministerial Decree number $65,07 / 03 / 2017$.
\end{tabular}

In the simplified method case, the determination of the risk classification is based on the European Macro seismic Scale (EMS), that allows for the identification of six different classes of vulnerability, according to the masonry structure typology ${ }^{14}$.

The Table 4 shows for each structure, the associated vulnerability class. In particular, the circle indicates the most reliable value, compared to the continuous and dashed lines which respectively refer to probable and less probable values. Thus a range is established in which the class of belonging is determined, for each individual structure.

12 The elements that are considered for the calculation of the expected average annual loss are direct economic losses and the annual average frequency of exceeding the events that seriously damage the building in question.

13 The index IS-V, also known as Risk Index, is determined through relevant and close evaluations, realized by specified experts, that allow to identify the value of PGA (Peak ground acceleration, that is equal to the maximum ground acceleration that occurred during earthquake shaking at a location), the place where there is the building and the construction conditions.

14 Based on the scale EMS-98. See European Council (1998). 
The evaluation of a certain level of dispersion is based on the status of the structure, as well as on the quality of the construction, which can affect the vulnerability in a more or less elevated way.

Here we refer to a series of considerations of a technical-construction nature, which require an accurate analysis carried out by experts who analyze the state of the buildings.

Table 4. Vulnerability class attribution for masonry constructions.



Source: Ministerial Decree number 65, 07/03/2017.

The elements, on which the examination is focused on, are referred to both external and internal structures.

Here we consider:

- The resistance of fundamentals and their relative rigidity;

- $\quad$ the binder quality;

- $\quad$ the possible presence of wooden frame;

- the material typology used for the construction;

- $\quad$ the constructive quality;

- $\quad$ the deterioration and/or the damaging;

- $\quad$ the wall height;

- $\quad$ every other element that can influence the construction evaluation.

With respect to the extent of the considered ranges, in order to improve building classification it is common to use statistical data linked to seismic events that, if they have to occur, might or might not damage the structure (Zuccaro 2004). As a consequence, it is necessary to adopt an interdisciplinary approach, combining statistical data with the evaluation of the structure typology, in order to obtain a classification as detailed and real as possible.

Concluding the analysis of evaluation and classification plans of buildings seismic exposition, the next step, provided by the new Decree, is based on the determination of a preventive plan, evaluating every intervention that can be realized with the goal of obtaining risk mitigation and the determination of the new exposition class. Every realized activity needs to be submitted to an accurate analysis, in order to evaluate the efficacy and the effectiveness in terms of structural improvements in relation to the risk exposition. 
Here we need to consider that each home could require specific interventions, which compared to others, may differ. For this reason, in order to identify the new classification of buildings, it is necessary to develop a specific examination that can certify the status pre and post intervention. In fact, each activity may be evaluated differently depending on the case in question.

Similar devices are adopted for industrial warehouses. Also in this case, indeed, the set of rules quoted above can be used. However, there is a significant difference from houses that allows for the simplification and speeding up of the process. In the case of industrial warehouse is sufficient to carry out reinforcement works for the structure and to follow the indicated instructions in order to obtain a low exposition to seismic phenomena, without being necessary to carry out a preventive evaluation.

As far as the evaluation and the certification of the realized interventions are concerned, the Decree states that these activities be carried out by qualified experts, all of whom have the required knowledge to do so.

False declarations represent, in this contest, a relevant risk, because they can attest a building state that is different from the real condition. They are frauds that can cause extremely serious consequences, not only for the building's inhabitants, but also for those in the nearby buildings.

In order to avoid such events, a "permanent monitoring commission" is established in the Decree, at the "Consiglio Superiore dei Lavori Pubblici". This Commission holds the role of supervisor with the task of examining and giving a judgment on the effectiveness of the prevention intervention, thus being able to validate or not the statements made by the experts.

This whole series of interventions is accompanied by a deduction system, with a duration of 5 years. This is a method by which an amount relative to the interventions carried out is subtracted from taxes due to the State.

In particular, the legislation presents a system of rewarding deductions. In other words, we can identify a model that allows for the evaluation of the amount of the deduction proportionally with respect to the intervention and the relative real improvement of the dwelling, in terms of risk classes.

Going into detail to determine the amount of the deductions, it is necessary to differentiate two different types: on the one hand housing (first or second house) and productive buildings, on the other condominiums and common parts.

The deduction amount in a proportional way respect to and to the relative real improvement of the home, in terms of risk classes.

As far as the first case is concerned, there is a deduction of $70 \%$ with respect to an improvement of one risk class. If, after the intervention, there is an improvement of two or more classes, the deduction will be equal to $80 \%$.

With regard to the second hypothesis, there is a deduction of $75 \%$ for an improvement of a risk class, or one of $85 \%$ for two or more classes.

The obtainment of such tax benefits can be only be achieved after the certification of the work carried out and their compliance with the project and with respect to the sector legislation.

Furthermore, it is expected that these measures be applied for a spending amount not greater than 96,000 euro per property unit, per year; in the case of joint ownerships, therefore, the amount of 96,000 euro is to be multiplied by the number of property units that form the entire building.

According to the Ministerial Decree, all the evaluations, quoted above, have to be reported in a certification, drawn up following the indications set in the enclosed B of the Decree. It is a conclusive operation that involves the completion of the entire procedure. It summarizes all the information linked to the evaluation of the works done, according to what has been declared by the expert instructed to certify the completed intervention. It serves to confirm the passage into a better risk class and, as a consequence, to allow owners to benefit from the relative tax concessions.

This provision opens up new scenarios of intervention, with a view to developing and disseminating methods of ex-ante contrast to damage deriving from environmental disasters. The possibility of using tax concessions for improvements to the buildings is an important and significant risk mitigation strategy. The more relevant the latter may be the more spread the Government strategy will be. 
To these obvious but necessary considerations, we need to add more about the concrete possibility of seeing economic policies materialize, which, together with the provision described in detail, can allow Italy to take a considerable leap in quality in terms of exposure to seismic risk.

\section{Conclusions}

The "Sisma Bonus" by Italian Government, as described in the article, is a relevant national plan of building prevention towards seismic events. Undoubtedly it is a measure that moves in the direction of a better citizens' consciousness of these themes. In a country, like Italy, where citizens refer almost only to the Government after a natural disaster, this measure has a relevant role to play trying to develop a different culture. In this sense, it could be a "trailblazer" towards new interventions.

Through this kind of interventions should be possible to spread a better consciousness about vulnerability given a detailed knowledge of all the elements that influence the exposition to natural catastrophes. The presence of tax deductions is fundamental to incentivize the interventions and allow a true application of these policy in Italy.

In this prospective, this article outlines that the effects of the "Sisma Bonus" would be to facilitate the private action, in the perspective of providing insurance instruments and solutions that can make Italy a country that is able to efficiently contrast the damages deriving from natural disasters.

Author Contributions: All authors have contributed significantly to this research in all phases and sections.

Conflicts of Interest: The authors declare no conflicts of interest.

\section{References}

Ania. 2017. Assicurazione Incendio Abitazioni Civili. Roma: Ania Trends, September.

Birkmann, Jörn. 2006. Measuring Vulnerability to Natural Hazards—Towards Disaster Resilient Societies. Tokyo: United Nations University Press.

Canuti, Paolo, and Nicola Casagli. 1994. Considerazioni sulla Valutazione del Rischio di Frana. Roma: Consiglio Nazionale delle Ricerche.

D'Ayala, Dina, Alex Copping, and Hui Wang. 2006. A conceptual model for multi-hazard assessment of the vulnerability of historic buildings. In Structural Analysis of Historical Constructions: Possibilities of Numerical and Experimental Techniques, Proceedings of the Fifth International Conference, New Delhi, India, 6-8 November. Boca Raton: CRC Press.

Einstein, H. H. 1988. Landslide risk assessment procedure, Special lecture. Paper presented at V International Symposium on Landslides, Lausanne, Switzerland, July 10-15, pp. 1075-90.

Eurisko. 2017. Apertura delle Famiglie Italiane Verso una Polizza casa a Copertura dei DANNI da Catastrofi Naturali. Milan: Survey GfK Eurisko, June.

European Commission. 2013. Green Paper on the Insurance of Natural and Man-Made Disasters. Strasbourg: European Commission, April.

European Council. 1998. Cahiers du Centre Européen de Géodynamique et de Séismologie. Edited by G. Grünthal. Luxembourg: European Macroseismic Scale, vol. 15.

European Environment Agency. 2010. Mapping the Impacts of Natural Hazards and Technological Accidents in Europe. An Overview of the Last Decade. Technical Report. Copenhagen: European Environment Agency.

Farina, Maria Bianca. 2017. Cosa Fanno le Assicurazioni per Prevenire i Rischi e Proteggere i Cittadini? Paper presented at ANIA, La giornata Internazionale per la riduzione dei Danni Catastrofali, Roma, Italy, October 13.

Fell, Robin. 1994. Landslide risk assessment and acceptable risk. Canadian Geotechnical Journal 31: $261-72$. [CrossRef]

Guidobboni, Emanuela, and Gianluca Valensise. 2013. L'Italia dei Disastri: Dati e Riflessioni sull'impatto Degli Eventi Naturali, 1861-2013. Bologna: Bononia University Press.

PERILS. 2017. PERILS Discloses Final Loss Estimate of EUR 108M for the August 2016 Earthquake in Central Italy. Press Release. Zurich: PERILS, August.

Porrini, Donatella. 2010. L'assicurazione sui disastri naturali: Motivi della scarsa diffusione e soluzioni di politica economica. Politica Economica 1: 123-45. 
Porrini, Donatella. 2016. Risk Classification in Natural Catastrophe Insurance: The Case of Italy. International Journal of Financial Research 7: 39. [CrossRef]

Porrini, Donatella, and Reimund Schwarze. 2014. Insurance models and European climate change policies: An assessment. European Journal of Law and Economics 38: 7-28. [CrossRef]

Ronchi, Romina. 2017. Rischio catastrofale e coperture assicurative: Le possibili soluzioni per l'Italia, Convegno: Il rischio da calamità naturali: Aspetti tecnici, giuridici ed assicurativi. In Focus sui sistemi di protezione civile locale e sulla gestione dei soccorsi. Novara: Associazione Nazionale fra le Imprese Assicuratrici.

Swiss Re. 2017. Economic Research E Consulting and Cat Perils. Zurich: Swiss Re.

Swiss Re Institute. 2017. Natural Catastrophes and Man-MADE Disasters in 2016: A Year of Widespread Damages. Sigma no 2/2017. Zurich: Swiss Re Institute.

UNDP. 2014. Human Development Report 2014-Sustaining Human Progress: Reducing Vulnerabilities and Building Resilience. New York: UNDP.

UNISDR. 2004. Living with Risk: A Global Review of Disaster Reduction Initiatives. Geneva: UNISDR.

Varnes, D.J. 1984. Landslides Hazard Zonation: A Review of Principles and Practice. Natural Hazards. Paris: UNESCO. Weymann, Martin. 2015. Natural Disasters Risk Management. Santiago de Chile: ASSAL Seminar.

Wisner, Ben, Piers Blaikie, Terry Cannon, and Ian Davis. 2003. At Risk: Natural Hazards, People's Vulnerability and Disasters, 2nd ed. Abingdon: Routledge.

Zuccaro, G. 2004. Inventario e Vulnerabilità del Patrimonio Edilizio Residenziale del Territorio Nazionale, Mappe di Rischio e Perdite Socio-Economiche. Napoli: CD-ROM.

(C) 2018 by the authors. Licensee MDPI, Basel, Switzerland. This article is an open access article distributed under the terms and conditions of the Creative Commons Attribution (CC BY) license (http:/ / creativecommons.org/licenses/by/4.0/). 